\section{Acute-onset Pretibial Swelling}

A 5-year-old girl presented with a rapidly growing, nontender swelling in her pretibial region. There was history of repetitive hits of the tricycle pedals on the shins. A solid subcutaneous mass was palpated. Laboratory tests were unremarkable. Magnetic-resonance-imaging showed a hyperintense sub-cutaneous mass and a hyperintense area consistent with bone marrow edema. As malignancy was a concern, excisional biopsy was performed. Histopathological examination revealed subcutaneous granuloma annulare (SGA).

SGA is a benign, inflammatory lesion occurring in otherwise healthy preschoolers in the lower extremity, especially the pretibial area, in hands, scalp and buttocks. The pathogenesis is unknown, the association with diabetes mellitus is controversial, and the description of triggering events like trauma has anecdotal interest. In this case, the repetitive trauma justified the associated bone marrow edema. The pediatricians should take care not to

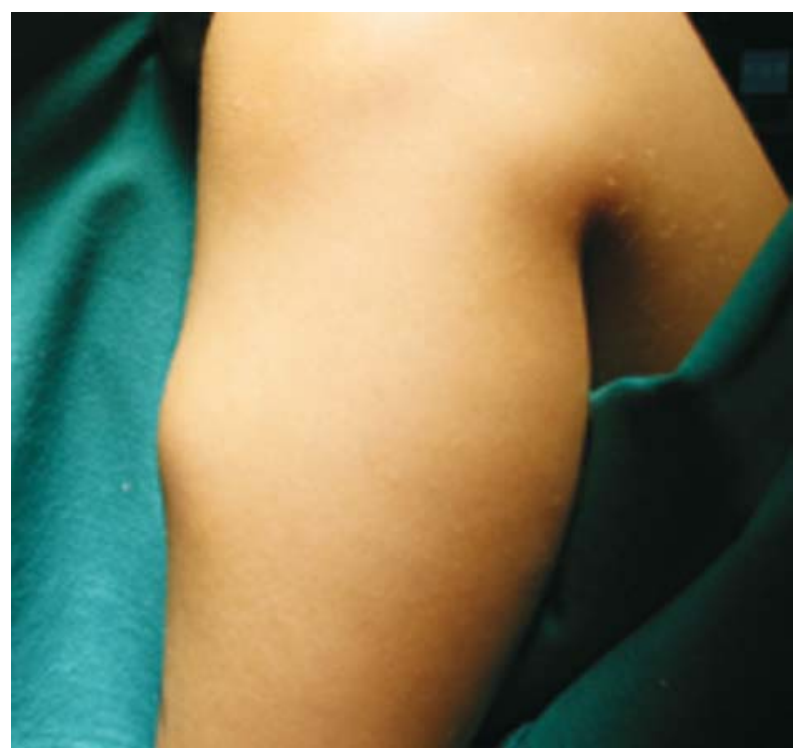

FIG. 1 Subcutaneous mass in the left pretibial region.

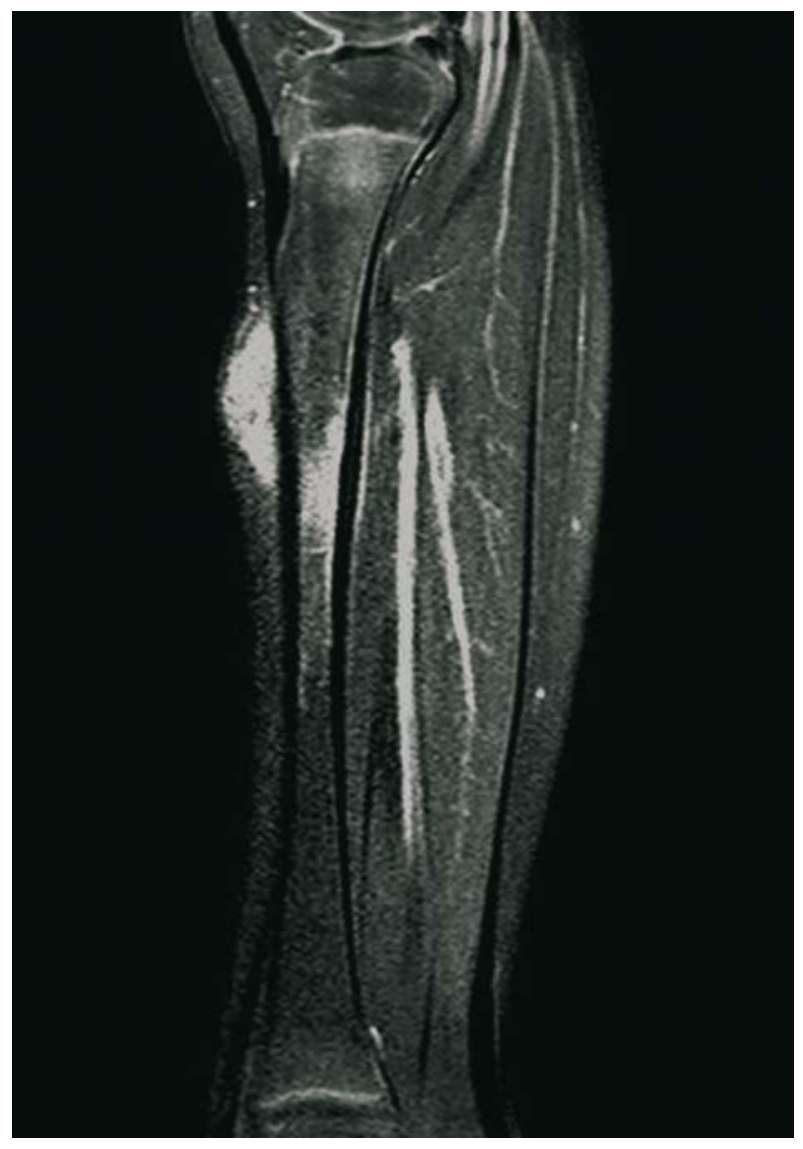

FIG. 2 T2-weighted sagittal MRI of the left pretibial region showing a hyperintense well-circumscribed mass extending to but not traversing the underlying fascia. A consensual area of hyperintensity of the bone marrow was seen.

misdiagnose these cases as malignancy, as we did, indicating unnecessary treatments while spontaneous regression is the rule.

SARA Lega, MARCo RABUSIN AND
*FEDERICA PEDERIVA
Departments of Hematology-Oncology and
*Pediatric Surgery,
Institute for Maternal and Child Health-
IRCCS “Burlo Garofolo”-Trieste, Italy.
federica_pederiva@yahoo.it

